\title{
RISK FACTORS OF COMPLICATED COMMUNITY-ACQUIRED PNEUMONIA IN CHILDREN: 1-YEAR DATA FROM A TERTIARY HEALTHCARE CENTER
}

\author{
Gokcen Tugcu ${ }^{1}$, Beste Ozsezen ${ }^{1}$, İrem Türkyılmaz ${ }^{1}$, Betül Zorlu${ }^{1}$, Sanem Eryılmaz ${ }^{1}$, \\ Aslinur Parlakay ${ }^{1}$, and Guzin Cinel $^{1}$ \\ ${ }^{1}$ Ankara City Hospital
}

May 11, 2021

\begin{abstract}
Introduction:Community-acquired pneumonia(CAP) in children has tended to decrease in recent years with effective vaccination and treatments. However, the frequency of complicated community-acquired pneumonia (CCAP) is gradually increasing.By determining risk factors for the development of CCAP,new approaches for early diagnosis and effective treatment can be determined. Method: Records of 113 patients who were hospitalized due to CAP and CCAP between January 2017 and December 2017 were examined.Demographic characteristics of the patients,comorbid diseases,admission symptoms,physical examination findings,laboratory and imaging results, treatments, hospital stay and treatment response were recorded. Pleural effusion,if empyema developed in patients with CCAP,thoracentesis and/or chest tube insertion,fibrinolytic therapy,and the need for additional surgical intervention were investigated. Results:Our patients had a mean age of $3.6 \pm 2.2,60(53 \%)$ were female and $53(47 \%)$ were male.93 $(82.3 \%)$ of the patients were diagnosed with CAP and 18(15.9\%) were diagnosed with CCAP.Detection of pleural effusion at the admission of patients in multivariate logistic regression[(OR (95\% CI),4.24 (1.77-10.16), p <0.001)] respiratory distress(tachypnea and retraction)[(OR (95\% CI) $3.04(1.30-7.130))$,p <0.001)] and hypoxia (92\% and less saO2 measured by pulse oximeter) at admission [(OR (95\% CI),: $5.247(1.58-9.46), \mathrm{p}<0.001)$ ] were identified as independent risk factors for diagnosis of CCAP. Discussion:Hypoxia,respiratory distress and imaging pleural effusion are important distinguishing findings for development of complications in patients admitted for CAP. Determining the etiology of CCAPs and early diagnosis and treatment approaches can be established, and protection measures can be taken. With future prospective studies, the causes of CCAP can be determined and preventive measures and new treatment approaches can be developed
\end{abstract}

\section{INTRODUCTION:}

The incidence of community-acquired pneumonia (CAP) is gradually decreasing due to appropriate antibiotic therapy, vaccination, and improvements in community health services ${ }^{1}$. CAP is one of the most important causes of morbidity and mortality particularly in children aged below 5 years ${ }^{2}$. The hospitalization rate of children with CAP is between $9.5 \%$ and $42 \%$, and the average hospitalization duration is 9 days $^{3}$. In $10 \%$ of hospitalized patients, CAP is accompanied by simple parapneumonic effusion (PPE) ([?]10 mm, without fibrous septation ${ }^{4,5}$. Regardless of the need for hospitalization, CAPs are associated with complicated community-acquired pneumonia (CCAP) with local complications, such as PPE, empyema (EMP), necrotizing pneumonia (NP), lung abscess (LA), and bronchopleural fistula (BPF) ${ }^{4}$ In approximately $7 \%$ of patients, CAP progresses to NP, characterized by consolidation and parenchymal necrosis ${ }^{6}$. Then, NP may rapidly worsen to cavitation (pneumatocele) with peripheral and single lobe involvement and BPF when the pleural space is affected; therefore, patients should be closely monitored for complications ${ }^{7}$.

CCAP causes include immune deficiencies, malnutrition, congenital cystic lung malformations, and foreign body aspiration (FBA). However, it is also observed in previously healthy children ${ }^{8}$. CCAP risk factors 
include prolonged fever before hospitalization; antipyretic, acetaminophen, and ibuprofen use; age $<2$ years; asymmetric chest pain during the initial admission; high acute phase reactant levels; leukopenia; and iron deficiency anemia ${ }^{9-11}$.

CAP and CCAP patients present with consolidation, atelectasis, cavitation, and effusion are observed on chest radiography (CXR). Hence, imaging can provide important information. However, consolidation may not be clearly observed on CXR during the early stages of $\mathrm{NP}^{7}$. Nevertheless, thoracic USG is performed to assess low-volume effusions that are overlooked on CXR. Therefore, it can be used as a guide during thoracentesis and GT insertion ${ }^{12}$. Thoracic computed tomography (CT) scan is conducted to obtain differential diagnosis including tumor and congenital airway malformations and to facilitate surgical planning for large pneumatoceles that are unresponsive to treatment, ${ }^{13}$. In addition, by appropriately identifying CT, LA, and NP, the duration, content and treatment type can be modified ${ }^{13}$.

BPF should be considered in NP patients who had persistent $(>24 \mathrm{~h})$ air leakage during follow-up ${ }^{14,15}$. LA is characterized by subfebrile fever and chest pain. Further, it has a less noisy clinic than NP and may cause pneumothorax similar to NP and BPF. However, mediastinal shift is rarely observed. NP and/or LA patients subsequently experience clinical and radiological improvements. In addition, surgical intervention is commonly required due to complications such as pneumothorax, BPF, and pneumatocele ${ }^{14-16}$.

With appropriate antibiotic therapy and supportive treatments, simple pleural effusion can improve in CAPs patients ${ }^{15}$ Due to local and systemic complications and surgical interventions (mainly NP and LA), CCAP patients require long-term antibiotic treatment ${ }^{15,17}$. NPs and LAs are managed with similar antibiotic protocols; however, as the healing process in LA is slow, it requires longer antibiotic treatment ${ }^{18}$.

Chest tube drainage is the primary approach for symptomatic patients, even in those with small pleural effusion and loculations ${ }^{18,19}$. If adequate pleural fluid drainage cannot be achieved with GT in septal pleural effusion and EMP patients, the hospitalization duration is shortened using fibrinolytics ${ }^{20}$. Video-assisted thoracic surgery (VATS) can be performed when adequate drainage cannot be achieved with $\mathrm{CDF}^{21}$. Since there is a BPF risk during CDF, particularly when accompanied by NP and LA, treatment should be discontinued immediately ${ }^{22}$. VATS in the early period is associated with a lower BPF risk in NPs with EMP and septal pleural effusion ${ }^{23,24}$. Aspiration and drainage can be applied if there is no clinical improvement with antibiotic therapy and if complications including mediastinal shift develop in LA patients. The risk of fistulation into the pleural space after drainage is lower than that of $\mathrm{NPs}^{24}$. However, advanced surgical intervention should be considered in large pneumatoceles that do not respond to conservative treatment for severe $\mathrm{BPF}^{6,25,26}$.

The current study aimed to examine the CCAP progression risk factors by assessing the clinical and laboratory characteristics of children with CAP and the treatment strategies used for managing complications in patients who are unresponsive to treatment.

\section{METHOD:}

Pneumonia was diagnosed based on clinical, laboratory, and radiological data. Its severity and the diagnosis of complicated pneumonia were confirmed via radiological examination. In the presence of PPE, NP, EMP, and LA, CCAP is considered. Simple pleural effusion accompanied by CAPs was treated, and thoracic USG was performed to assess effusion ([?]10 mm, without septations).

\section{Patient data :}

Data about demographic characteristics such as age and sex; admission symptoms; physical examination findings; laboratory and imaging results; treatment information in the file records of 113 patients aged between 1 month and 18 years who were hospitalized in our hospital's pediatric wards between January 01, 2017, and December 31, 2017, due to CAP and CCAP; and treatment response were obtained. Moreover, information regarding comorbidities (such as asthma, immunodeficiency, and congenital heart disease) and treatments was investigated. Medical history, duration of fever, and antibiotic therapy history was noted. In patients diagnosed with CCAP who developed PPE and EMP, data about medical procedures such 
as thoracentesis and/or chest tube insertion, pleural fluid examination, microbiological analysis, and additional imaging; surgical intervention (thoracentesis, GT, and VATS), and hospitalization duration were investigated. Patients with an underlying chronic lung disease (including cystic fibrosis and bronchiectasis), hospital-acquired/ventilator-associated pneumonia, primary or secondary immunodeficiency, and swallowing dysfunction were excluded from the study. Patients were divided into two groups (those without pulmonary complications and those without). Then, the two groups were compared using the abovementioned variables.

\section{Statistical Analysis And Ethics}

The Statistical Package for the Social Sciences software version 25.0 (IBM Corp., Armonk, NY, the USA) was used for data analysis. Variables were expressed as mean \pm standard deviation, percentage, and frequency. Categorical data were analyzed using the Fisher's exact test and the chi-square test. $\mathrm{P}<0.05$ and $<0.01$ were acceptable. The possible risk factors identified in the univariate analyses were further used in the multivariate analysis to determine the independent predictors of CCAP. For subgroup analyses, unadjusted odds ratio (OR) and relative $95 \%$ confidence interval (CI) were used, and a p[?]0.005 was considered significant.

Ethics approval:

The local institutional review board approved this study (2019-179).

\section{RESULTS:}

\section{Demographic and clinical findings:}

In total, $60(53 \%)$ patients were women and $53(47 \%)$ men. The mean age of the participants was 3.6 +- 1.2 years (range: 3 months to 18 years). Thirty (26.5\%) patients had a body weight below the 10th percentile for age. Moreover, 93 (82.3\%) were diagnosed with CAP and 18 (15.9\%) with CCAP. There was no significant difference in sex between the two groups. In total, $15(13 \%)$ patients had a history of previous CAP. Further, during admission, 105 (92.9\%) had cough; 91 (80.5\%), fever; and 8 (7.07\%), chest pain. Then, $43(38.05 \%)$ patients received peroral antibiotic-antiviral therapy $(\mathrm{n}=12$, amoxicillin-clavulanic acid; $\mathrm{n}=8$, cephalosporins; $\mathrm{n}=14$, macrolides; $\mathrm{n}=7$, amoxicillin-clavulanic and macrolides; and $\mathrm{n}=2$, oseltamivir) before admission. Antibiotic use before admission and history of pneumonia did not significantly differ between the CAP and CCAP groups $(\mathrm{p}=0.250,0.223,0.324,0.659,0.253$, and 0.546 , respectively). The average CCAP patients' age was $4.2+-3.3$ years. The age of CAP patients was greater than the mean age $(2.8+-2.1$ years $)(\mathrm{p}=0.012)$. In our study, none of the patients presented with severe malnutrition. Although patients diagnosed with CCAP commonly have a body weight below the 10th percentile for age, the 2 groups did not significantly differ $(\mathrm{p}=0.024)$. Respiratory distress (tachypnea and retraction) and hypoxia $(\mathrm{SaO} 2<92 \%)$ were significantly more common in patients diagnosed with CCAP at presentation than in those diagnosed with CAP ( $\mathrm{p}<0.001$ and $<0.001$, respectively) (Table-1). None of the CAP and CCAP patients died due to local and systemic complications during hospitalization (Table-1).

\section{Hospitalization and supportive treatments}

In total, 16 (14.1\%) patients required follow-up in the pediatric intensive care unit (PICU). Moreover, 56 $(49.5 \%)$ received oxygen therapy via nasal cannula (NC), and $18(15.9 \%)$ via high-frequency nasal cannula (HFNC). Thirteen (11.5\%) patients were treated with noninvasive mechanical ventilation (NIMV) and 2 $(1.8 \%)$ with invasive mechanical ventilation (IMV). In the CCAP group, hypoxia upon admission and the need for inhaled oxygen via NC during hospitalization were more common in the CCAP group than in the CAP group $(\mathrm{p}<0.001$ and $<0.001$, respectively). CCAP patients commonly required HFNC $(\mathrm{p}=0.01)$. Admission at PICU and need for NIMV were more common in CCAP patients than in CAP patients $(\mathrm{p}<0.001$ and $<0.001$, respectively). Meanwhile, the mean hospitalization durations in CAP and CCAP patients were $6.35+-2.41$ and $19.71+-15.60$ days, respectively. Therefore, the two groups significantly differed in terms of hospitalization duration $(\mathrm{p}<0.001)$ (Table-2). No patient developed systemic complications including sepsis, shock, and multiple organ failure.

\section{Comorbidities:}


Fifteen (13\%) patients diagnosed with asthma/wheezing were previously followed-up. Of 113 patients, 2 were followed-up for prematurity, 1 for congenital hypothyroidism, and 1 for gastroesophageal reflux disease (GERD). During hospitalization, three $(2.7 \%)$ patients were diagnosed with selective IgA deficiency $(\mathrm{n}=1$, CCAP and $n=2, C A P)$, and one $(0.9 \%)$ of three children presented with transient hypogammaglobulinemia. One patient presented with ASD on echocardiogram (ECHO) and one with PDA based on the presence of heart murmur. Hence, comorbidities are not significant risk factors for the development of CCAP $(\mathrm{p}=0.243)$ (Table-1).

Due to secondary pulmonary hypertension (PHT) on ECHO, inhaled iloprost was administered to one patient who received IMV in the PICU due to CCAP and who was followed-up. With appropriate antibiotic therapy and respiratory support, PHT improved; thus, treatment with inhaled iloprost was discontinued. Two patients were diagnosed with GERD during hospitalization based on clinical and radiological findings, and their complaints improved with appropriate treatment during follow-up. One patient with FBA who was followed-up for CCAP and whose anamnesis was suspicious underwent rigid bronchoscopy, and YC was removed. One patient with GT implantation who received TPA due to PPE and NO developed pneumatocele. However, the condition improved with appropriate antibiotic therapy during follow-up. One patient who received treatment for LA presented with BPF, and surgery was performed because the patient was symptomatic. Nevertheless, none of the patients underwent lobectomy.

\section{Imaging:}

All patients underwent CXR during hospitalization. The incidence of consolidation on CXR was similar in the CCAP and CAP groups. However, effusion was more common in CCAP patients than in CAP patients $(\mathrm{p}<0.001)$. CCAP patients often present with atelectasis $(\mathrm{p}=0.027)$ and pneumothorax $(\mathrm{p}=0.01)$. Simple pleural effusion was observed on CXR, and three (3.22\%) CAP patients underwent thoracic USG. Moreover, $13(72.2 \%)$ CCAP patients with suspicious CXR and other findings upon admission underwent CT scan because sufficient information could not be obtained via thoracic USG. Then, $3(3.22 \%)$ of 93 CAP patients were followed-up for persistent fever and CCAP.

Of 18 patients diagnosed with CCAP, 8 (44.43\%) presented with PPE, 7 (38.88\%) with EMP, $2(11.1 \%)$ with NP, and $1(5.55 \%)$ with LA. One patient who was followed-up due to CCAP presented with Morgagni hernia on CT scan, and surgical consultation was then requested. One patient with NP had pneumatocele. In addition, another patient who was followed-up for LA had BPF (Table-3).

\section{Microbiological analysis:}

The hemoglobin level, thrombocyte and leukocyte count, CRP level, and erythrocyte sedimentation rate (ESR) were examined upon admission, and there was no significant difference in terms of these factors between the CCAP and CAP groups. CCAP patients had a significantly higher CRP level than CAP patients $(10.06+-7.55$ vs $4.43+-3.37 \mathrm{~g} / \mathrm{L}, \mathrm{p}=0.007)$ (Table-1). No pathological growth was observed in the samples in one patient who underwent sputum culture and in 15 patients who underwent thoracentesis. In $3(4.4 \%)$ of 68 patients who underwent blood culture, growth was observed. In two patients who were followed-up for CAP, growth in the blood culture during follow-up ( $\mathrm{n}=1$, Staphylococcus SPP and $n=1$, Streptococcus acidominimus ) was considered contamination, and there was no growth in the control blood cultures. Moreover, clinical and radiological improvements were observed in these patients without changing the current therapy. Pseudomonas aeruginosa growth was observed in the blood culture of a patient who was followed for CCAP and pneumatocele after NP, and treatment was modified accordingly (Table-4).

In total, $22(19.46 \%)$ patients underwent respiratory viral DNA polymerase chain reaction (PCR) amplification, and microorganisms were not detected via PCR in 8 patients. In total, 14 (63.6\%) patients $(\mathrm{n}=6$, respiratory syncytial virus [RSV]; $\mathrm{n}=3$, adenovirus; $\mathrm{n}=2$, influenza type $\mathrm{A} ; \mathrm{n}=1$, coronavirus; $\mathrm{n}=1$, metapneumovirus; and $n=1$, bocavirus) had positive PCR results. Not all patients can undergo respiratory viral PCR amplification due to technical problems. Hence, RSV and other viral agents identified via viral PCR amplification were not significant risk factors for CCAP development (Table-4). 


\section{Treatments:}

In total, 66 (70.6\%) of 93 hospitalized CAP patients received intravenous sulbactam ampicillin (SAM). Of these patients, $24(25.8 \%)$ were treated with simultaneous peroral macrolide. Further, of 18 CCAP patients, $16(88.8 \%)$ received intravenous vancomycin and cefotaxime; $6(33.3 \%)$, clindamycin; and $3(16.66 \%)$, peroral macrolide. Three $(3.22 \%)$ CAP patients underwent CT scan in addition to thoracic USG due to fever lasting $>3$ days and clinical deterioration. Then, treatment was modified in CCAP patients. The initial treatment protocols significantly differed between the two groups $(\mathrm{p}<0.001)$ (Table-4).

\section{Procedures:}

In total, 11 of 15 PPE and EMP patients underwent thoracentesis. Two patients had pleural glucose levels $<40 \mathrm{mg} / \mathrm{dL}$. The mean pleural lactate dehydrogenase level was $1825.48+-1484.00 \mathrm{U} / \mathrm{L}$. A chest tube (GT) was inserted in $9(50 \%)$ patients. Moreover, $4(26.66 \%)$ patients who had fibrous septations on thoracic USG received TPA at an average of $2.60+-1.14$ times. The mean chest tube duration was $9.10+-6.3$ days. One patient with a GT inserted in another hospital who did not have enough pleural fluid during follow-up but who had septal effusion with intense content on thoracic USG was treated with VATS. Further, he was followed-up 5 days after surgery and was discharged on the 10th day of admission.

\section{Independent variables:}

In the multivariate logistic regression analysis, pleural effusion, respiratory distress (tachypnea and retraction), and hypoxia at presentation were considered independent risk factors for CCAP (Table-5).

\section{DISCUSSION:}

In the current study, patients diagnosed with CCAP at presentation were older than those diagnosed with CCAP. Sex and comorbidities were not significant risk factors for CCAP. Meanwhile, age below 2 years, male sex, and comorbidities were associated with invasive pneumococcal infections ${ }^{16,27,28}$. However, age, sex, and comorbidities were not significant risk factors for pneumococcal pneumonia. In a study conducted in Israel, there was no significant difference in age between CAP and CCAP patients. Moreover, age $<2$ years was not a risk factor for CCAP development ${ }^{29}$. Immunodeficiencies and suppurative lung diseases may cause CAP. However, CCAPs are generally observed in children who do not have comorbidities and who were previously healthy ${ }^{29}$. In a 540 patient series in the USA in 2002, children diagnosed with EMP were older (mean age $=6$ years, $\mathrm{p}<0.001$ ) than those who were followed-up for $\mathrm{CAP}^{30}$. In a study that assessed the 10-year medical records of patients in Canada and France, CCAP was found to account for $51 \%-57 \%$ of all hospital admissions among children aged below 5 years ${ }^{28,31}$ Similar findings were also observed in a study including 767 patients (mean age $=4.1$ years, $\mathrm{p}=0.02$ ) in France in 2010,31.

In this study, the presence of cough, chest pain, and fever and pre-admission time of these symptoms and a history of pneumonia were not considered risk factors for CCAP. Respiratory distress (tachypnea and retraction) and hypoxia were significantly associated with CCAP. Severe CAP is defined as the presence of fever; capillary filling time $>2 \mathrm{~s}$; dehydration; inability to feed; subcostal, nasal, and substernal retraction; tachypnea (infants, $>70$ cycles $/ \mathrm{min}$ and older children, $>50$ cycles $/ \mathrm{min}$ ); cyanosis; and moaning, according to the British Thoracic Society 2011 guideline ${ }^{4}$. Based on these parameters, CCAP is more common in hospitalized patients diagnosed with severe pneumonia ${ }^{4,5}$.

In our study, CCAP patients were commonly underweight. Even in the absence of severe malnutrition, underweight is associated with an increased pneumonia risk ${ }^{32}$. Wexler ID et al. conducted a study in Israel. Results showed that CCAP patients were often underweight, and this finding is similar to ours. However, the condition is not an independent risk factor for CCAP development ${ }^{9}$.

In our study, CCAP patients had a high CRP level at time of enrollment. Moreover, both groups had a high ESR and leukocyte and thrombocyte counts during admission. In the study of Huang C-Y et al., elevated CRP level and leukocyte count upon admission were a significant factor for CCAP development ${ }^{10}$. In a multicenter study that retrospectively analyzed 111 pneumonia patients in 2006, significant differences in 
terms of ESR and leukocyte and platelet counts were not observed between CAP and CCAP patients ${ }^{9}$. In these patient groups, anemia and leukocyte $<15.000 \mathrm{~mm}^{3}$ were associated with prolonged fever and longer hospitalization duration ${ }^{9}$.

In our study, no significant growth was observed in the blood cultures of patients hospitalized due to CAP and CCAP. This was attributed to peroral antibiotic use before hospitalization. Regardless of the presence of pulmonary complications, the bacteremia incidence is low in patients followed-up for pneumonia, and a possible pathogen is rarely detected in the blood culture ${ }^{11,33}$. Neuman MI et al. conducted a study in the USA in 2017. Results showed that a pathogenic microorganism was detected in the blood culture in only $65(2.5 \%)$ of 2568 patients hospitalized due to pneumonia ${ }^{33}$. In this study, empirical antibiotic use before hospitalization less likely caused growth in the blood culture ${ }^{33}$. In a retrospective study conducted by Erlichman I et al., which included 144 children hospitalized due to CCAP, empirical antibiotics use before hospitalization decreased the growth rate in the blood culture from $63 \%$ to $22 \%$. However, the pathogen proportion detected in pleural cultures was higher ${ }^{34}$. In a 20 -year retrospective study in the USA, the blood culture growth rate decreased from $67 \%$ to $30 \%$ with empirical antibiotic use before hospitalization in 369 patients who had EMP ${ }^{35}$. In a study examining patients who were followed-up for PPE and EMP between 2010 and 2017, the pathogen proportion was significantly lower in the blood culture than in pleural cultures $^{33}$.

Viral and bacterial PCR amplification of samples collected from the respiratory tract and pleura could not be obtained from each patient due to technical problems. RSV was the most common viral agent detected. Within the last 10 years, respiratory and pleural fluid DNA PCR amplification methods, which can yield faster results than conventional culture and are not affected by empirical antibiotic use, are commonly used in pathogen identification ${ }^{9,20,36}$. The DNA PCR of samples collected from pleural fluid can provide more sensitive results than that of blood culture samples, and the serotyping results can be obtained significantly faster ${ }^{36}$. However, there are limitations in the differentiation of pathogenic microorganisms due to colonization when nasopharyngeal, oropharyngeal, sputum samples are used ${ }^{37}$.

In our study, according to CT scan data, the antibiotic therapy type and duration was modified and more detailed information could be obtained before the surgical intervention, particularly in our patient with NP and BPF. If fever does not subside within $72 \mathrm{~h}$ and the clinical condition does not improve or worsens in patients followed-up for CAP, treatment for CCAP should be modified, if necessary, according to the evaluation results ${ }^{21,38}$. When CXR and thoracic USG are not effective for the diagnosis of complications, such as BPF and pneumatocele, particularly in the differentiation of NP and LA, more detailed information can be obtained via $\mathrm{CT} \operatorname{scan}^{22,38}$

In our study, pleural effusion $>10 \mathrm{~mm}$ during admission is a significant variable for CCAP diagnosis. Similar studies have shown that the presence of pleural effusion at presentation is associated with CCAP diagnosis ${ }^{9-11}$. In patients with unresolved radiological and clinical findings, YCA, tuberculosis-related lymphadenopathy, and congenital lung malformations may be observed.

In accordance with studies in the literature, our research showed that CCAP patients commonly present with hypoxia upon admission, and oxygen therapy was required during hospitalization. CCAP patients have a longer hospitalization duration than CAP patients due to local and systemic complications and the need for additional interventions (GT, CDF, and VATS) during follow-up ${ }^{39}$. BPF was observed, with an incidence rate of $13 \%$ in children with NP in the USA and $67 \%$ in Brazil ${ }^{12,14}$. Patients followed-up for CCAP, particularly NP and BPF with complications, had a longer hospitalization, oxygen therapy, and fever duration and higher number of PICU and even ECMO follow-ups ${ }^{16,40}$.

In our study, intravenous SAM and/or peroral macrolide were administered to patients diagnosed with CAP, and treatment was modified when CCAP was detected. The antibiotic duration and type used before admission were not significant risk factors for CCAP development. In previous retrospective studies and a prospective research conducted by Huang CY et al., disease progression was observed regardless of the empirical antibiotic therapy type provided according to the current treatment guidelines ${ }^{10,15}$. 
VATS is associated with better outcomes and shorter hospitalization duration compared with pleural drainage with GT without fibrinolytics ${ }^{20}$. In 2021, the American Academy of Pediatric Surgery recommended that VATS should be considered when chemical debridement with CDF cannot be achieved ${ }^{20}$. In a systematic review and meta-analysis published in 2018, similar perioperative complications were observed in cases in which both methods were used. However, patients who received VATS were exposed to less repetitive interventions and had a shorter hospitalization duration ${ }^{41}$. Based on these data, in recent years, studies about early VATS use, instead of CDF, in children have been published in accordance with those in adults ${ }^{42}$. When sufficient debridement with GT is not achieved and VATS is performed in the early period before fibrinolytic use, the hospitalization duration is shortened. Further, the complication incidence is lower in patients treated with VATS than in those treated with $\mathrm{CDF}^{42}$. Based on these developments, VATS is preferred instead of CDF when adequate pleural fluid drainage cannot be achieved with thoracentesis and GT implantation for PPE and EMP treatment in our hospital after 2017, which was the time our study was conducted.

The surgical intervention timing can affect treatment success ${ }^{43,44}$. CDF can be used for early-stage disease management. However, it is not effective for advanced-stage disease, and complication incidence increases with repeated interventions including CDF. Moreover, hospitalization duration is prolonged ${ }^{43}$. VATS efficacy decreases, particularly after 1 month, and decortication may be required in patients who underwent $\mathrm{CDF}^{43-46}$. In relation to this, the European Association for Cardio-Thoracic Surgery recommends that VATS should be highly considered in all advanced-stage disease patients at presentation ${ }^{46}$

The current study had several limitations. That is, the research was retrospective in nature. Hence, antipyretic treatment use, content, and frequency, as well as its effect on CCAP development, could not be investigated because the patients' family members did not regularly follow-up for fever. Moreover, our hospital is a tertiary referral center, and most patients had received prior treatment. However, late referrals could affect outcomes. Although necessary, not all patients could undergo respiratory and pleural DNA PCR amplification due to technical problems.

In conclusion, pleural effusion, respiratory distress (tachypnea and retraction), and hypoxia at presentation were found to be independent risk factors for CAP. Therefore, experience in interventional radiology and pediatric surgery and use of multidisciplinary approaches may be important for the diagnosis and treatment of children with CCAP admitted in tertiary care centers. However, multicenter, prospective and comparative studies should be conducted to examine CDF and VATS efficacy in children with early- and late-stage diseases.

\section{References:}

1. Bowen SJ, Thomson AH. British Thoracic Society Paediatric Pneumonia Audit: a review of 3 years of data. Thorax. 2013 Jul;68(7):682-3.

2. GBD 2016 Lower Respiratory Infections Collaborators. Estimates of the global, regional, and national morbidity, mortality, and aetiologies of lower respiratory infections in 195 countries, 1990-2016: a systematic analysis for the Global Burden of Disease Study 2016. Lancet Infect Dis 2018; 18: 1191210.

3. Atkinson M, Lakhanpaul M, Smyth A, Vyas H, Weston V, Sithole J et al. Comparison of oral amoxicillin and intravenous benzyl penicillin for community acquired pneumonia in children (PIVOT trial): a multicentre pragmatic randomised controlled equivalence trial. Thorax. 2007 Dec;62(12):1102-6.

4. Harris M, Clark J, Coote N, Fletcher P, Harnden A, McKean M, Thomson A; British Thoracic Society Standards of Care Committee. British Thoracic Society guidelines for the management of community acquired pneumonia in children: update 2011. Thorax. 2011 Oct;66 Suppl 2:ii1-23.

5. Clark JE, Hammal D, Spencer D, Hampton F. Children with pneumonia: how do they present and how are they managed? Arch Dis Child. 2007 May;92(5):394-8.

6. Masters IB, Isles AF, Grimwood K. Necrotizing pneumonia: an emerging problem in children? Pneumonia (Nathan) 2017; 9: 11.

7. Gross I, Gordon O, Cohen-Cymberknoh M, Reiter J, Tsabari R, Gileles-Hillel A et al. Giant lung cysts 
following necrotizing pneumonia: Resolution with conservative treatment. Pediatr Pulmonol. 2019 Jun;54(6):901-906.

8. Lahti E, Peltola V, Virkki R, Alanen M, Ruuskanen O. Development of parapneumonic empyema in children. Acta Paediatr. 2007 Nov;96(11):1686-92.

9. Wexler ID, Knoll S, Picard E, Villa Y, Shoseyov D, Engelhard D, Kerem E. Clinical characteristics and outcome of complicated pneumococcal pneumonia in a pediatric population. Pediatr Pulmonol. 2006 Aug;41(8):726-34.

10. Huang CY, Chang L, Liu CC, Huang YC, Chang LY, Huang YC et al; Taiwan Pediatric Infectious Disease Alliance. Risk factors of progressive community-acquired pneumonia in hospitalized children: a prospective study. J Microbiol Immunol Infect. 2015 Feb;48(1):36-42.

11. Le Bourgeois M, Ferroni A, Leruez-Ville M, Varon E, Thumerelle C, Bremont F, et al. Children, Antibiotics, Nonsteroidal Anti-inflammatory Drugs and Childhood Empyema (ChANCE) Study Group. Nonsteroidal Anti-Inflammatory Drug without Antibiotics for Acute Viral Infection Increases the Empyema Risk in Children: A Matched Case-Control Study. J Pediatr. 2016 Aug;175:47-53.e3.

12. Lewis MR, Micic TA, Doull IJM, Evans A. Real-time ultrasound-guided pigtail catheter chest drain for complicated parapneumonic effusion and empyema in children - 16-year, single-centre experience of radiologically placed drains. Pediatr Radiol. 2018 Sep;48(10):1410-1416.

13. Jaffe A, Calder AD, Owens CM, Stanojevic S, Sonnappa S. Role of routine computed tomography in paediatric pleural empyema. Thorax 2008; 63: 897-902.

14. Sawicki GS, Lu FL, Valim C, Cleveland RH, Colin AA. Necrotising pneumonia is an increasingly detected complication of pneumonia in children. Eur Respir J 2008; 31: 1285-91.

15. Bradley JS, Byington CL, Shah SS, Alverson B, Carter ER, Harrison C, et al. Pediatric Infectious Diseases Society and the Infectious Diseases Society of America. Executive summary: the management of community-acquired pneumonia in infants and children older than 3 months of age: clinical practice guidelines by the Pediatric Infectious Diseases Society and the Infectious Diseases Society of America. Clin Infect Dis. 2011 Oct;53(7):617-30.

16. Hsieh YC, Wang CW, Lai SH, Lai JY, Wong KS, Huang YC et al. Necrotizing pneumococcal pneumonia with bronchopleural fistula among children in Taiwan. Pediatr Infect Dis J. 2011 Sep;30(9):740-4.

17. Ross RK, Hersh AL, Kronman MP, Newland JG, Metjian TA, Localio AR, Zaoutis TE, Gerber JS. Impact of Infectious Diseases Society of America/Pediatric Infectious Diseases Society guidelines on treatment of community-acquired pneumonia in hospitalized children. Clin Infect Dis. 2014 Mar;58(6):8348.

18. de Benedictis FM, Carloni I. Management of necrotizing pneumonia in children: Time for a patientoriented approach. Pediatr Pulmonol. 2019 Sep;54(9):1351-1353.

19. Balfour-Lynn IM, Abrahamson E, Cohen G, Hartley J, King S, Parikh Det al.; Paediatric Pleural Diseases Subcommittee of the BTS Standards of Care Committee. BTS guidelines for the management of pleural infection in children. Thorax. 2005 Feb;60 Suppl 1(Suppl 1):i1-21.

20. Islam S, Calkins CM, Goldin AB, Chen C, Downard CD, Huang EY,et al.; APSA Outcomes and Clinical Trials Committee, 2011-2012. The diagnosis and management of empyema in children: a comprehensive review from the APSA Outcomes and Clinical Trials Committee. J Pediatr Surg. 2012 Nov;47(11):2101-10.

21. Segerer FJ, Seeger K, Maier A, Hagemann C, Schoen C, van der Linden M, et al. Therapy of 645 children with parapneumonic effusion and empyema-A German nationwide surveillance study. Pediatr Pulmonol. 2017 Apr;52(4):540-547.

22. Long AM, Smith-Williams J, Mayell S, Couriel J, Jones MO, Losty PD.'Less may be best'-Pediatric parapneumonic effUsion and empyema management: Lessons from a UK center. J Pediatr Surg. 2016;51: 588-591.

23. Kalfa N, Allal H, Lopez M, Counil FO, Forgues D, Guibal MP, Galifer RB. An early thoracoscopic approach in necrotizing pneumonia in children: a report of three cases. J Laparoendosc Adv Surg Tech A. $2005 \mathrm{Feb} ; 15(1): 18-22$.

24. Hoffer FA, Bloom DA, Colin AA, Fishman SJ. Lung abscess versus necrotizing pneumonia: implications 
for interventional therapy. Pediatr Radiol. 1999 Feb;29(2):87-91.

25. Nagasawa KK, Johnson SM. Thoracoscopic treatment of pediatric lung abscesses. J Pediatr Surg 2010; 45: $574-78$.

26. Dugan KC, Laxmanan B, Murgu S, Hogarth DK. Management of persistent air leaks. Chest 2017; 152: $417-23$

27. Kelly MM, Coller RJ, Kohler JE, Zhao Q, Sklansky DJ, Shadman KA, et al.Trends in Hospital Treatment of Empyema in Children in the United States. J Pediatr. 2018 Nov;202:245-251.e

28. Langley JM, Kellner JD, Solomon N, Robinson JL, Le Saux N, McDonald J,et al. Empyema associated with community-acquired pneumonia: a Pediatric Investigator's Collaborative Network on Infections in Canada (PICNIC) study. BMC Infect Dis. 2008 Sep 25;8:129.

29. Wexler ID, Knoll S, Picard E, Villa Y, Shoseyov D, Engelhard D, Kerem E. Clinical characteristics and outcome of complicated pneumococcal pneumonia in a pediatric population. Pediatr Pulmonol. 2006 Aug;41(8):726-34.

30. Byington CL, Spencer LY, Johnson TA, Pavia AT, Allen D, Mason EO, et al.An epidemiological investigation of a sustained high rate of pediatric parapneumonic empyema: risk factors and microbiological associations. Clin Infect Dis. 2002 Feb 15;34(4):434-40.

31. Francois P, Desrumaux A, Cans C, Pin I, Pavese P, Labarere J. Prevalence and risk factors of suppurative complications in children with pneumonia. Acta Paediatr. 2010 Jun;99(6):861-6.

32. Huysentruyt K, Alliet P, Raes M, Willekens J, De Schutter I, De Wachter E,et al. Nutritional status of children hospitalized for parapneumonic effusion. PLoS One. 2014 Apr 4;9(4):e94242.

33. Neuman MI, Hall M, Lipsett SC, Hersh AL, Williams DJ, Gerber JS, et al. Pediatric Research in Inpatient Settings Network. Utility of Blood Culture Among Children Hospitalized With CommunityAcquired Pneumonia. Pediatrics. 2017 Sep;140(3):e20171013.

34. Erlichman I, Breuer O, Shoseyov D, Cohen-Cymberknoh M, Koplewitz B, Averbuch D, et al. Complicated community acquired pneumonia in childhood: Different types, clinical course, and outcome. Pediatr Pulmonol. 2017 Feb;52(2):247-254.

35. Stankey CT, Spaulding AB, Doucette A, Hamre KES, Wheeler W, Pomputius WF, Kurachek S. Blood Culture and Pleural Fluid Culture Yields in Pediatric Empyema Patients: A Retrospective Review, 1996-2016. Pediatr Infect Dis J. 2018 Sep;37(9):952-954.

36. Krenke K, Sadowy E, Podsiadły E, Hryniewicz W, Demkow U, Kulus M. Etiology of parapneumonic effusion and pleural empyema in children. The role of conventional and molecular microbiological tests. Respir Med. 2016 Jul;116:28-33.

37. Lahti E, Peltola V, Waris M, Virkki R, Rantakokko-Jalava K, Jalava J, Eerola E, Ruuskanen O. Induced sputum in the diagnosis of childhood community-acquired pneumonia. Thorax. 2009 Mar;64(3):252-7.

38. Andronikou S, Goussard P, Sorantin E. Computed tomography in children with community-acquired pneumonia. Pediatr Radiol. 2017 Oct;47(11):1431-1440.

39. Liese JG, Schoen C, van der Linden M, Lehmann L, Goettler D, Keller S,et al. Changes in the incidence and bacterial aetiology of paediatric parapneumonic pleural effusions/empyema in Germany, 2010-2017: a nationwide surveillance study. Clin Microbiol Infect. 2019 Jul;25(7):857-864.

40. Stroud MH, Okhuysen-Cawley R, Jaquiss R, Berlinski A, Fiser RT. Successful use of extracorporeal membrane oxygenation in severe necrotizing pneumonia caused by Staphylococcus aureus Pediatr Crit Care Med 2007; 8: 282-87.

41. Pacilli M, Nataraja RM. Management of paediatric empyema by video-assisted thoracoscopic surgery (VATS) versus chest drain with fibrinolysis: systematic review and meta-analysis. Paediatr Respir Rev 2019; 30: 42-48.

42. Victora CG, Kirkwood BR, Ashworth A, Black RE, Rogers S, Sazawal S, Campbell H, Gove S. Potential interventions for the prevention of childhood pneumonia in developing countries:improving nutrition. Am J Clin Nutr. 1999 Sep;70(3):309-20.

43. Goldin AB, Parimi C, LaRiviere C, Garrison MM, Larison CL, Sawin RS. Outcomes associated with type of intervention and timing in complex pediatric empyema. Am J Surg. 2012 May;203(5):665-673.

44. Li ST, Gates RL. Primary operative management for pediatric empyema: decreases in hospital length 
of stay and charges in a national sample. Arch Pediatr Adolesc Med. 2008 Jan;162(1):44-8.

45. James CA, Braswell LE, Pezeshkmehr AH, Roberson PK, Parks JA, Moore MB. Stratifying fibrinolytic dosing in pediatric parapneumonic effusion based on ultrasound grade correlation. Pediatr Radiol. 2017 Jan;47(1):89-95.

46. Scarci M, Abah U, Solli P, Page A, Waller D, van Schil P, et al. EACTS expert consensus statement for surgical management of pleural empyema. European journal of cardio-thoracic surgery : official journal of the European Association for Cardio-thoracicSurgery. 2015;48(5):642-53.

\section{Hosted file}

Table-1 Clinical and demographic findings in community-acquired pneumonia (CAP) and complicated communi available at https://authorea.com/users/369107/articles/521670-risk-factors-of-complicatedcommunity-acquired-pneumonia-in-children-1-year-data-from-a-tertiary-healthcare-center

\section{Hosted file}

Table-2 Radiology, supportive treatments and hospitalization duration in CAP and CCAP.pdf available at https://authorea.com/users/369107/articles/521670-risk-factors-of-complicatedcommunity-acquired-pneumonia-in-children-1-year-data-from-a-tertiary-healthcare-center

\section{Hosted file}

Table-3Microbiological results and antibiotic therapy in CAP and CCAP.pdf available at https://authorea.com/users/369107/articles/521670-risk-factors-of-complicated-communityacquired-pneumonia-in-children-1-year-data-from-a-tertiary-healthcare-center

\section{Hosted file}

Table-4 Independent risk factors in CCAP development based on multivariate logistic regression.pdf available at https://authorea.com/users/369107/articles/521670-risk-factors-of-complicatedcommunity-acquired-pneumonia-in-children-1-year-data-from-a-tertiary-healthcare-center 


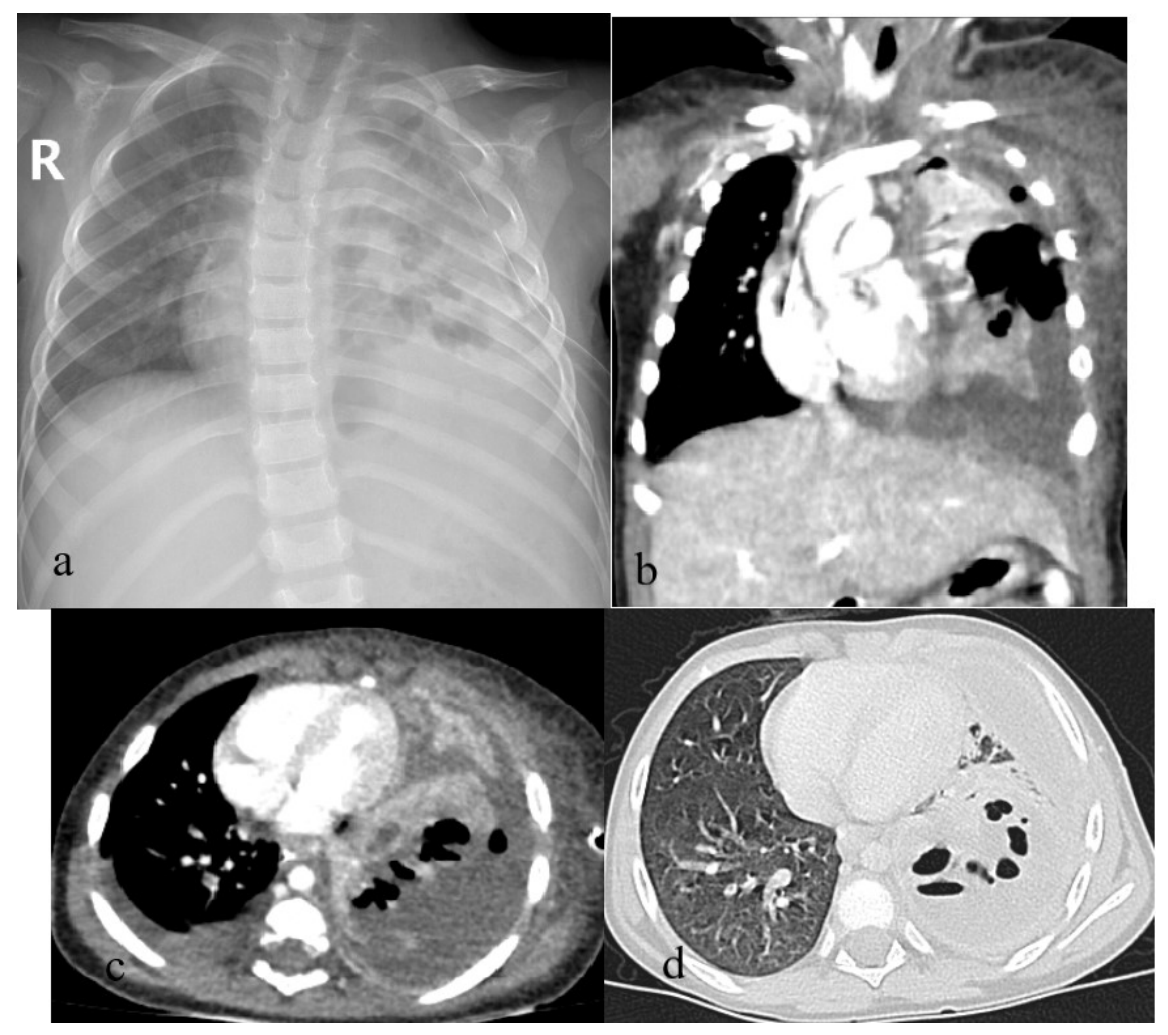




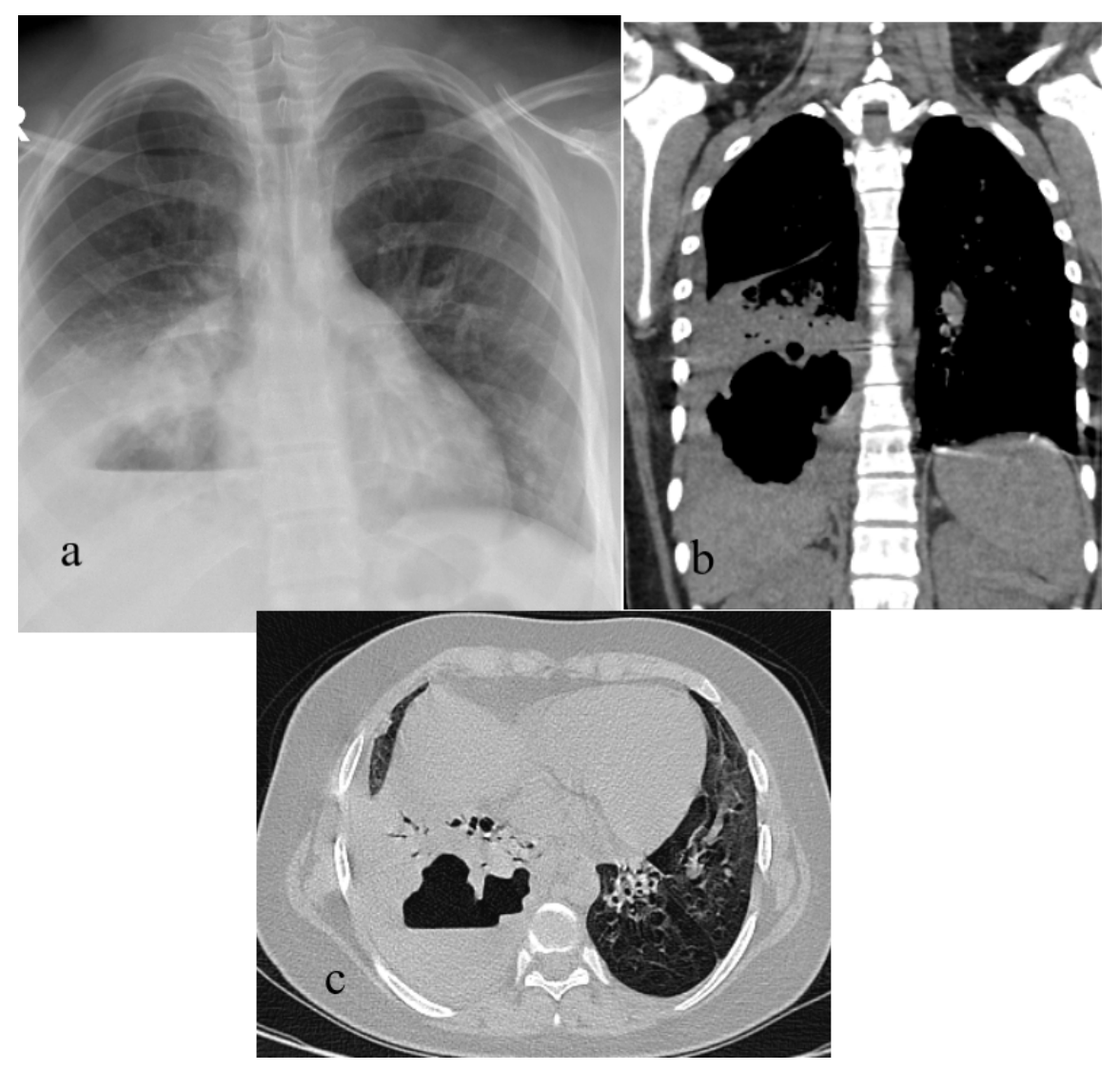

